\title{
Combined treatment of malignant salivary gland tumours with intensity-modulated radiation therapy (IMRT) and carbon ions: COSMIC
}

\author{
Alexandra D Jensen ${ }^{1 *}$, Anna Nikoghosyan ${ }^{1}$, Christine Windemuth-Kieselbach², Jürgen Debus ${ }^{1}$, Marc W Münter ${ }^{1}$
}

\begin{abstract}
Background: Local control in malignant salivary gland tumours is dose dependent. High local control rates in adenoid cystic carcinomas could be achieved by highly conformal radiotherapy techniques and particle (neutron/ carbon ion) therapy. Considering high doses are needed to achieve local control, all malignant salivary gland tumours probably profit from the use of particle therapy, which in case of carbon ion treatment, has been shown to be accompanied by only mild side-effects.

Methods/design: The COSMIC trial is a prospective, mono-centric, phase II trial evaluating toxicity (primary endpoint: mucositis $\geq \mathrm{CTCAE}^{\circ} 3$ ) and efficacy (secondary endpoint: local control, disease-free survival) in the combined treatment with IMRT and carbon ion boost in 54 patients with histologically proved ( $\geq$ R1-resected, inoperable or $\mathrm{Pn+}$ ) salivary gland malignancies. Patients receive $24 \mathrm{GyE}$ carbon ions (8 fractions) and IMRT (50 Gy at $2.0 \mathrm{~Gy} /$ fraction).

Discussion: The primary objective of COSMIC is to evaluate toxicity and feasibility of the proposed treatment in all salivary gland malignancies.
\end{abstract}

Trial Registration: Clinical trial identifier NCT 01154270

\section{Background}

Malignant salivary gland tumours are rare and a heterogenous group of tumours accounting for about 3-5\% of head and neck cancers. High-grade tumours such as mucoepidermiod carcinoma (35\%) and adenoid cystic carcinoma (25\%) are the most common histological subtypes [1], characterised by a rather slow pattern of growth, perineural spread and high propensity for haematogenous metastases. So far, standard therapy of high-grade salivary gland carcinoma consists of complete surgical resection and adjuvant radiation in highrisk situations $(\mathrm{R}+$ or close margin, perineural spread, neural infiltration, large tumours (T3/4), or nodal metastases [2-4]. To achieve local control, radiation doses of $>60$ Gy or even 66 Gy (5) are recommended $[5,6]$, with apparently all tumour stages profiting from postoperative radiotherapy [5,7-9].

\footnotetext{
* Correspondence: alexandra.jensen@med.uni-heidelberg.de

'Dept. of Radiation Oncology, INF 400, 69120 Heidelberg, Germany

Full list of author information is available at the end of the article
}

Regarding radiation therapy, local control was also significantly improved by the application of high-precision techniques, dose-escalation and high-LET radiation [9-13].

Intensity-modulated radiation therapy (IMRT) as well as fractionated stereotactic RT could already improve local control as compared to conventional RT techniques achieving 3-year PFS rates of 38\% [14].

So far, the highest local control rates at $75-100 \%$ $[11,13]$ were achieved by neutron radiation albeit at the cost of significant late toxicity.

Dose escalation [12] though only in a small group of patients, in treatment with heavy ions at $70.2 \mathrm{GyE}(3 \times$ $3.9 \mathrm{GyE} / \mathrm{wk})$ or $64 \mathrm{GyE}(4 \times 4 \mathrm{GyE} / \mathrm{wk})$ was shown to be feasible and beneficial. Despite high doses per fraction, no CTC ${ }^{\circ} \mathrm{III}$ late toxicities and very few ${ }^{\circ} \mathrm{III}$ acute reactions occurred. Local control at 5 years was still 100\%, however, various histological subtypes of salivary gland tumours were included in the trial. Pommier et al. [15] treated 23 patients with adenoid cystic carcinoma with protons at 75,9 GyE (median) in various fractionation 
schemes. Overall local control at 5 years was 93\%, however, the authors noted several ${ }^{\circ} \mathrm{III}$ as well as one ${ }^{\circ} \mathrm{V}$ late toxicity (temporal lobe necrosis). Douglas et al [11] published a retrospective analysis of 159 patients with adenoid cystic carcinoma treated with neutrons (total dose: 19,2 Gy), but only reported somewhat disappointing local control rates of $57 \%$ at 5 years accompanied with significant late toxicity $\left(14 \%>\mathrm{CTC}^{\circ} \mathrm{III}\right)$. Results of the combined IMRT-C12 treatment therefore compare favourably [10] with only very mild treatment-related side effects ( ${ }^{\circ} \mathrm{III}$ late tox in only 1 patient) [16,17]. 54 Gy

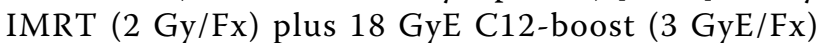
yielded local control rates of about $78 \%$ at 4 years [10].

In view of the outcome and low toxicity profile, photon IMRT plus C12-boost has been accepted as the standard treatment and method of choice for adenoid cystic carcinoma in Germany. Due to the possibility to apply higher doses at so far mild side effects, chargedparticle therapy does promise improved results for all types of malignant salivary gland cancers.

Therefore, this trial basically evaluates relative dose escalation with simultaneous normal tissue sparing by using a combination of C12-heavy ion therapy and IMRT in all salivary gland malignancies including mucoepidermoid carcinoma and adenoid cystic carcinoma. Looking at other head and neck tumors i.e. squamous cell carcinoma of the head and neck (SCCHN), radiation therapy commonly causes pronounced mucositis. With therapy usually about 7 weeks duration and mucositis, improvement of clinical symptoms can only be expected at least 2-3 weeks post completion of therapy. At the occurrence of mucositis CTC ${ }^{\circ} \mathrm{III}$, pain, loss of taste, and dysphagia causing reduced food/fluid intake and weight loss can potentially cause treatment delays, protraction of treatment and worse outcome. Therefore, evaluation of this particular side effect was chosen as the primary endpoint of the trial. Trial objectives hence assess accompanying acute and late toxicity and focusing on mucositis CTC ${ }^{\circ}$ III. Secondary endpoints consist of evaluation of efficacy (local control, disease-free survival) and toxicity (other than mucositis).

Initial results of the pilot project at the GSI/Darmstadt (treatment of skull base ACC) reported mucositis CTC ${ }^{\circ}$ III or higher in only $6 \%$ of cases, with a recent update confirming these findings [18]. Assuming higher normal tissue sparing by increase of the heavy-ion part of the treatment, we would expect toxicity/mucositis rates unchanged or even lower.

\section{Methods/design}

\section{Study design}

The COSMIC trial is a prospective, non-randomized phase II feasibility trial evaluating acute mucositis $\geq$ CTCAE ${ }^{\circ}$ III (v. 3.0) as the primary endpoint. Mucositis
CTCAE ${ }^{\circ}$ III is defined as confluent ulcerations or pseudomembranes within the oral cavity and bleeding with minor trauma leading to the inability of the patient to adequately aliment or hydrate orally.

\section{Study Characteristics}

Combined IMRT and C12-heavy ion boost has previously been established as a treatment of choice in skull-base adenoid cystic carcinoma. However, based on the fact that local control seems to be dose-dependent, all types of salivary gland malignancies in the head and neck will probably benefit from high-LET radiation. Therefore, the combination of IMRT (50 Gy in 2 Gy/ fraction) and C12-boost (24 GyE in $3 \mathrm{GyE} /$ fraction) will be tested as to toxicity profile and efficacy.

\section{Study objectives}

To evaluate feasibility and toxicity (with a focus on mucositis CTCAE ${ }^{\circ}$ III (v. 3.0)) and efficacy of the treatment.

Incidence of mucositis $\geq \mathrm{CTCAE}^{\circ} \mathrm{III}$ (v. 3.0) will be assessed as the primary endpoint of the trial, local control, disease-free survival, toxicity (incl. mucositis CTCAE ${ }^{\circ} \mathrm{I}-\mathrm{II}$ and late toxicity at 3 years post RT)

\section{Sample size/number of subjects}

Incidence of mucositis CTCAE ${ }^{\circ}$ III in the initial data was $6 \%$ for adenoid cystic carcinoma mostly of the skull base. 29 patients with adneoid cystic carcinoma were treated with 54 Gy IMRT and 18 GyE carbon ions, two of these patients (i.e. 6.9\%) showed mucositis CTCAE ${ }^{\circ}$ III. Inclusion and exclusion criteria in COS$\mathrm{MIC}$ correspond to the initial patient selection; hence incidence of toxicity should also roughly correspond. Agreeing to an incidence of $6 \%, 49$ patients have to be treated in order to observe at least one patient with mucositis CTCAE ${ }^{\circ} \mathrm{III}$ or higher with a probability of $95 \%$. Assuming a drop-out rate of $10 \%, 54$ patients need to be recruited.

\section{Patient selection}

Inclusion criteria

- Histologically confirmed or surgically removed malignant tumour of the salivary glands (head and neck)

- Inoperable tumour

- G2/3

- Macroscopic or microscopic residual tumour (R2/ R1) or

- $\geq \mathrm{T} 3 / \mathrm{T} 4$ or

- perineural invasion $(\mathrm{Pn}+)$

- written informed consent

- pts aged 18 - 80 years

- effective contraception for pts in childbearing age (< 12 months post beginning of menopause) 


\section{Exclusion criteria}

- Prior radio- or chemotherapy for tumours of the head and neck

- Other previous malignancy within the past 5 years except prior, adequately treated basal cell carcinoma of the skin or pre-invasive carcinoma of the cervix

- Significant neurological or psychiatric condition including dementia or seizures or other serious medical condition prohibiting the patient's participation in the trial by judgement of the investigators

- Legal incapacity or limited legal capacity

- Positive serum/urine $\beta$-HCG/pregnancy

- Drug abuse

\section{Radiotherapy}

\section{Immobilisation/planning examinations}

Patients are immobilized using individual thermoplastic head masks with thermoplastic shoulder fixation. Planning examinations consist of a planning CT scan $(3 \mathrm{~mm}$ slice thickness) with the patient positioned in the individual fixation device and contrast-enhanced MRI for 3D image correlation.

\section{Target volumes/dose prescription}

CTV1 (carbon ion boost) includes the macroscopic tumour/prior tumour bed with special focus on the R1area as well as respective neural pathways to the base of skull (cave: perineural invasion and skip lesions). For tumours of the parotid gland, the whole former parotid area is also included in the CTV1, if possible the mandibular joint is kept outside the CTV1. PTV1 consists of a $3 \mathrm{~mm}$ margin around the CTV1 but does not extend into critical organs at risk (i.e. brain stem, spinal cord).

We prescribe a dose of $24 \mathrm{GyE}$ carbon ions in $3 \mathrm{GyE} /$ fraction (5 fractions per week) to the CTV1, we aim at covering the CTV1 with the 95\% prescription isodose. Treatment is given at the HIT (Heidelberg ion therapy centre) after inverse treatment planning in active beam application (raster-scanning method). Daily image guidance consists of orthogonal $\mathrm{x}$-ray controls in treatment position.

CTV2 includes CTV1 with safety margins along typical pathways of spread. Only ipsilateral nodal levels (II and III) are includes, however, in case the primary tumour is/was located at midline or crossing midline, we cover bilateral nodal levels II and III. In case there is a pathological lymph node involvement, additional nodal level will be covered as indicated. CTV2 also encompasses the complete surgical operational area. The CTV2 also takes account for set-up variations, hence corresponds to the PTV2 (CTV2 = PTV2). Should the primary tumour be located within the parotid gland, also the parotid duct needs to be within the CTV2.

50 Gy IMRT (inversely planned step-and-shoot or tomotherapy technique) in 25 fractions ( 5 fractions per week) are prescribed to the CTV2 (coverage at least with the $90 \%$ prescription isodose) taking into account doses applied by daily image guidance with MV-cone-beam CT.

\section{Supportive therapy}

Patients do not routinely receive prophylactic feeding tubes, however if they uncommonly experience significant weight loss we will of course offer feeding tube insertion or parenteral feeding.

\section{Treatment schedule/follow-up}

After obtaining written informed consent, patients are included into the trial and receive their treatment planning investigations. Treatment usually starts with 8 fractions carbon ion $(8 \times 3$ GyE C12) therapy followed by $27 / 28$ fractions of IMRT corresponding to a total dose of approximately 74 GyE. Treatment duration is approximately 6-7 weeks (figure 1).

First follow-up examination including diagnostic, contrast-enhanced MRI will be carried out 6 weeks post completion of radiation treatment. Further controls including MRI are 3, 6, and 12 months thereafter, in 6 monthly intervals until 2 years post RT, then in yearly intervals. Abdominal ultrasound will be carried out q6 months, chest-CT q12 months. At each followup appointment, patients receive a symptom-oriented clinical examination, also patients' performance state (Karnofsky-Index), therapy-associated side effects as well as potential intercurrent therapy is recorded.

Patients are also encouraged to undergo regular check-ups incl. full ENT clinical examinations in regular intervals.

\section{Proteomics and Genomics}

For the proteomic examinations $30 \mathrm{~mL}$ venous blood will be collected from each subject prior to RT, at day 29 , day 44 or 45 , and at the $1^{\text {st }}$ and $2^{\text {nd }}$ follow-up visit. Thus, the overall volume of blood samples used for Proteomic/Genomic investigations will be approximately $200 \mathrm{~mL}$. Following parameters/pathways will be investigated:

- In order to predict the efficacy of the therapy blood will be collected during therapy and follow-up to detect and correlate the levels of well known tumour- and angiogenesis markers (VEGF, TGFAlpha, bFGF, IL8, k-ras, etc.) using Enzyme-Linked Immunosorbent Assay (ELISA).

- Further, platelet protein content (i.e. tumor angiogenesis growth factors and cytokines) will be analyzed using citrate blood samples and correlated with serum- and plasma- protein results.

In order to perform the genomic analysis, patients' blood samples are collected as indicated and RNA, miRNA and DNA isolation will be performed. Based on an established platform, linear RNA-amplification, 


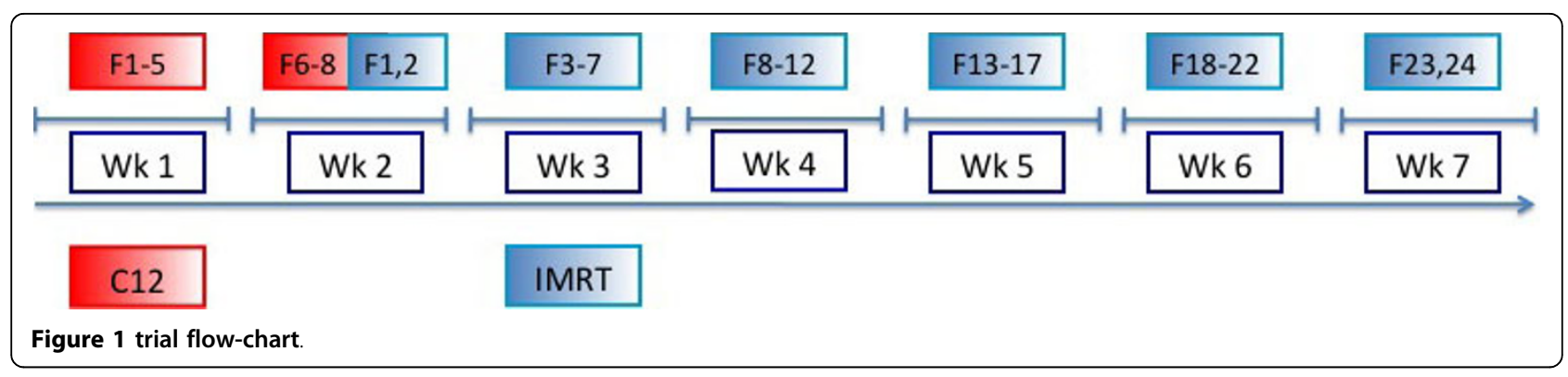

labelling and hybridization on human genome wide oligo-arrays (transcriptome analysis) are planned. DNA samples are used to identify potential chromosomal aberrations or epigenetic alterations that might predict treatment response. RNA and miRNA samples are further analyzed by real time quantitative RT-PCR to confirm microarray data and to test a subset of clinical predictors.

The determinations of proteomic and genomic parameters will be carried out at the Department of Radiation Oncology in Heidelberg. No further genetic investigations on the blood collected during the study will be carried out.

\section{Assessment of efficacy}

Assessment of efficacy will be carried out by evaluation of imaging studies (MRI) at each follow-up. If applicable (in case of initial macroscopic tumour) tumour response will be evaluated according to the RECIST-criteria (19).

\section{Trial organization/coordination}

The COSMIC trial has been designed by the Department of Radiation Oncology, University of Heidelberg, and is carried out at the Heidelberg Ion Therapy Centre (HIT). It is an investigator-initiated trial; the Department of Radiation Oncology is responsible for co-ordination, overall trial management, registration (clinicaltrials.gov Identifier: NCT 01154270), database management, quality assurance, monitoring, and reporting.

\section{Investigators}

Patients are recruited by the Department of Radiation Oncology.

\section{Adverse events}

Adverse and serious adverse events are recorded using NCI common toxicity criteria for adverse events (CTCAEAE v. 3.0). Acute radiation effects are defined as effects occurring within 90 days from beginning of radiotherapy. Late effects are defined as effects observed thereafter. Safety analysis is performed with respect to frequency of serious adverse events and adverse events stratified by organ system, severity, causality.

\section{Regular completion of the trial}

Patient accrual is completed with inclusion of the last patient and should extend for approximately 2 years from trial initiation. Regular trial participation for each patient terminates 3 years post inclusion into the trial or the patient's death respectively.

\section{Discontinuation of treatment}

- Patient wish

- Medical condition necessitating treatment termination and withdrawal of the patient from the trial

- Pregnancy

- Lack of compliance

\section{Premature termination of the trial}

The trial can be prematurely closed or suspended by the LKP in following cases:

- Medical or ethical reasons relevantly affecting the risk-benefit relationship,

- Difficulties in recruitment of subjects suggest unjustifiable prolongation of the study timeline,

- Previously unexpected adverse events (in respect of their nature, severity, duration or outcome) occur with unjustifiable frequency,

- Expected adverse events occur with an unexpectedly high incidence,

- Relevant superiority of patients in one treatment arm of a comparable clinical trial,

- Legal authorities' decision

The Ethics Committee (EC) and the competent regulatory authorities will be informed about premature closure of the trial. Furthermore, the Ethics Committee(s) and competent regulatory authorities themselves may decide to stop or suspend the trial.

If the trial is closed prematurely, the trial material such as completed, partially completed, and blank CRFs will be returned to the coordinating investigator.

All involved investigators have to be informed immediately about a cessation or suspension of the trial. The decision is binding on all trial centers and investigators.

\section{Ethics, informed consent, and safety}

The final protocol was approved by the ethics committee of the University of Heidelberg Medical School (S-263/2009). The trial complies with the Helsinki Declaration in its recent German version, the Medical Association's professional code of conduct, principles of Good Clinical Practice (GCP) guidelines and the Federal Data Protection Act. It will be carried out in keeping with local legal and regulatory requirements. It is also 
subject to authorization by the German radiation protection authority (Bundesamt für Strahlenschutz: $=$ BfS). Medical confidentiality and Federal Data Protection Act will be followed. Written informed consent is obtained from each patient in oral and written form.

\section{Discussion}

Achieving local control in malignant salivary gland tumour remains challenging. Progress has been made by the introduction of novel radiotherapy techniques like fractionated stereotactic RT and IMRT, however, particle therapy (neutron and carbon ion therapy) result in the highest control rates in adenoid cystic carcinoma so far. Compared to neutron RT, carbon ion treatment comes at the cost of only mild side effects and is now permanently available at a new dedicated particle therapy facility (HIT). Considering the fact local control in salivary gland tumours is dose dependent, we believe treatment outcome can be improved for all malignant salivary gland histologies without increasing side-effects by a combination of IMRT ( 50 Gy) with a carbon ion boost $(24 \mathrm{GyE})$ not only in incompletely resected or unresectable tumours but also in the R1-situation or perineural infiltration. The COSMIC trial was designed to address these issues in a prospective phase II trial. To our knowledge this is the first phase II trail combining particle therapy and IMRT.

\section{Author details}

${ }^{1}$ Dept. of Radiation Oncology, INF 400, 69120 Heidelberg, Germany. ${ }^{2}$ Alcedis GmbH, Winchester-Str. 2, 35394 Gießen, Germany.

\section{Authors' contributions}

ADJ, MWM,$C W K$, and JD developed the study protocol and planned the trial. CWK is responsible for statistical considerations/basis of the trial. ADJ, AN, MWM are responsible for conducting and co-ordination of the trial as well as patient recruitment. All authors read and approved the final manuscript

\section{Competing interests}

The authors declare that they have no competing interests.

Received: 9 July 2010 Accepted: 11 October 2010

Published: 11 October 2010

\section{References}

1. Spiro RH: Salivary neoplasms: overview of a 35-year experience with 2,807 patients. Head Neck Surg 1986, 8(3):177-84.

2. Chen AM, Granchi PJ, Garcia J, Bucci MK, Fu KK, et al: Local-regional recurrence after surgery without postoperative irradiation for carciomas of the major salivary glands: implications for adjuvant therapy. Int J Radiat Oncol Biol Phys 2007, 67:982-987.

3. Gurney TA, Eisele DW, Weinberg V, Shin E, Lee N: Adenoid cystic carcinoma of the major salivary glands treated with surgery and radiation. Laryngoscope 2005, 115(7):1278-82.

4. Mendenhall WM, Morris CG, Amdur RJ, Werning JW, Hinerman RW, Villaret DB: Radiotherapy alone or combined with surgery for adenoid cystic carcinoma of the head and neck. Head Neck 2004, 26(2):154-62.

5. Chen AM, Bucci MK, Weinberg V, Garcia J, Quivey JM, Schechter NR, et al: Adenoid cystic carcinoma of the head and neck treated by surgery with or without postoperative radiation therapy: prognostic features of recurrence. Int J Radiat Oncol Biol Phys 2006, 66(1):152-9.

6. Garden AS, Weber RS, Ang KK, Morrison WH, Matre J, Peters LJ: Postoperative radiation therapy for malignant tumors of minor salivary glands. Outcome and patterns of failure. Cancer 1994, 73(10):2563-9.

7. Terhaard CHJ, Lubsen H, Van der Tweel I, Hilgers FJM, Eijkenboom WMH, Marres HAM, et al: Salivary gland carcinoma: independent prognostic factors for locoregional control, distant metastases, and overall survival: results of the Dutch head and neck oncology cooperative group. Head Neck 2004, 26:681-92, discussion 692-3.

8. Terhaard CHJ, Lubsen H, Rasch CRN, Levendag PC: Kaanders HHAM, HjhoHaslinga $\mathrm{RE}$, et al. The role of radiotherapy in the treatment of malignant salivary gland tumors. Int J Radiat Oncol Biol Phys 2005, 61:103-111.

9. Bittner N, Koh WJ, Laramore GE, Patel S, Mulligan MS, Douglas JG: Treatment of locally advanced adenoid cystic carcinoma of the trachea with neutron radiotherapy. Int J Radiat Oncol Biol Phys 2008, 72:410-4.

10. Schulz-Ertner D, Nikoghosyan A, Didinger B, Munter M, Jakel O, Karger CP, et al: Therapy strategies for locally advanced adenoid cystic carcinomas using modern radiation therapy techniques. Cancer 2005, 104(2):338-44.

11. Douglas JG, Koh WJ, Austin-Seymour M, Laramore GE: Treatment of salivary gland neoplasms with fast neutron radiotherapy. Arch Otolaryngol Head Neck Surg 2003, 129(9):944-8.

12. Mizoe JE, Tsujii H, Kamada T, Matuoka Y, Tsuji H, Osaka Y, et al: Dose escalation study of carbon ion radiotherapy for locally advanced headand-neck cancer. Int J Radiat Oncol Biol Phys 2004, 60(2):358-64.

13. Huber PE, Debus J, Latz D, Zierhut D, Bischof M, Wannenmacher M, et al: Radiotherapy for advanced adenoid cystic carcinoma: neutrons, photons or mixed beam? Radiother Oncol 2001, 59(2):161-7.

14. Münter MW, Schulz-Ertner D, Hof H, Nikoghosyan A, Jensen A, Nill S, et al: Inverse planned stereotactic intensity modulated radiotherapy (IMRT) in the treatment of incompletely and completely resected adenoid cystic carcinomas of the head and neck: initial clinical results and toxicity of treatment. Radiat Oncol 2006, 1(17).

15. Pommier P, Liebsch NJ, Deschler DG, Lin DT, Mclntyre JF, Barker FG, et al: Proton beam radiation therapy for skull base adenoid cystic carcinoma. Arch Otolaryngol Head Neck Surg 2006, 132(11):1242-9.

16. Schulz-Ertner D, Nikoghosyan A, Jäkel O, Haberer T, Kraft G, Scholz M, et al: Feasibility and toxicity of combined photon and carbon ion radiotherapy for locally advanced adenoid cystic carcinomas. Int J Radiat Oncol Biol Phys 2003, 56:391-398.

17. Schulz-Ertner D, Nikoghosyan A, Thilmann C, Haberer T, Jäkel O, Karger C, et al: Results of carbon ion therapy in 152 patients. Int J Radiat Oncol Biol Phys 2004, 58:631-640.

18. Münter $M$, Umathum $V$, Nikoghosyan $A$, Jensen $A$, Hof $H$, Jaekel $O$, et al: Combination of intensity modulated radiation therapy (IMRT) and a carbon ion boost for subtotal resected or inoperable adenoid cystic carcinomas (ACC's) of the head and neck. PTCOG meeting 2009, abstract FC84.

19. Therasse P, Arbuck SG, Eisenhauer EA, Wanders J, Kaplan RA, Rubinstein L, et al: New guidelines to evaluate the response to treatment in solid tumors. J Nat/ Cancer Inst 2000, 92:205-216.

\section{Pre-publication history}

The pre-publication history for this paper can be accessed here: http://www.biomedcentral.com/1471-2407/10/546/prepub

doi:10.1186/1471-2407-10-546

Cite this article as: Jensen et al:: Combined treatment of malignant salivary gland tumours with intensity-modulated radiation therapy (IMRT) and carbon ions: COSMIC. BMC Cancer 2010 10:546. 